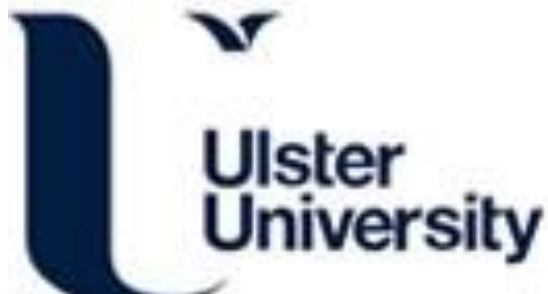

Elastic antibacterial membranes comprising particulate laden fibers for wound healing applications

Li, Y., Zhang, C., Zhu, L. F., Ahmad, Z., Li, J. S., \& Chang, M. W. (2019). Elastic antibacterial membranes comprising particulate laden fibers for wound healing applications. Journal of Applied Polymer Science, 136(8), [47105]. https://doi.org/10.1002/app.47105

Link to publication record in Ulster University Research Portal

\section{Published in:}

Journal of Applied Polymer Science

Publication Status:

Published (in print/issue): 20/02/2019

DOI:

10.1002/app.47105

\section{Document Version}

Author Accepted version

\section{General rights}

Copyright for the publications made accessible via Ulster University's Research Portal is retained by the author(s) and / or other copyright owners and it is a condition of accessing these publications that users recognise and abide by the legal requirements associated with these rights.

\section{Take down policy}

The Research Portal is Ulster University's institutional repository that provides access to Ulster's research outputs. Every effort has been made to ensure that content in the Research Portal does not infringe any person's rights, or applicable UK laws. If you discover content in the Research Portal that you believe breaches copyright or violates any law, please contact pure-support@ulster.ac.uk. 


\title{
Elastic Anti-Bacterial Membranes Comprising Particulate Laden Fibers for Wound Healing Applications
}

Yudong $\mathrm{Li}^{\mathrm{a}}$, Chunchen Zhang ${ }^{\mathrm{a}, \mathrm{b}}$, Li-Fang Zhu ${ }^{\mathrm{a}, \mathrm{b}}$, Zeeshan Ahmad ${ }^{\mathrm{c}}$, Jing-Song $\mathrm{Li}^{\mathrm{a}}$, Ming-Wei Chang ${ }^{\mathrm{a}, \mathrm{b}, *}$

a. Key Laboratory for Biomedical Engineering of Education Ministry of China, Zhejiang University, Hangzhou, 310027, P. R. China.

b. Zhejiang Provincial Key Laboratory of Cardio-Cerebral Vascular Detection Technology and Medicinal Effectiveness Appraisal, Zhejiang University, Hangzhou, 310027, P. R. China.

c. Leicester School of Pharmacy, De Montfort University, The Gateway, Leicester LE1 9BH, UK.

\begin{abstract}
Medicated skin care products are available in various forms; ranging from lotions and creams to bandages and membranes. In this study, anti-bacterial particulate laden fibrous membranes were prepared via electrospraying of tetracycline hydrochloride (TE-HCL) loaded poly( $\varepsilon$-caprolactone) (PCL) particles alongside electrospinning of thermoplastic polyurethane (TPU) fibers, through which both mechanical and biological aspects of a complete membrane system can be achieved. Random (R) and ordered (P and V) patterns of TPU fibrous membranes (FMs) were afforded using a rotating collector. Water contact angle and bacterial inhibition zone tests were performed to assess suitability of the system specifically for wound care. Stress-strain and in-vitro drug release tests were performed to assess suitability of newly developed systems specifically for hybrid membranes (HMs). The highest tensile strength $(32.1 \pm$ $4.9 \mathrm{MPa})$ with elasticity $(104.2 \pm 6.0 \%)$ and the most sustained release rate indicate HMs (P) are potentially suitable materials for wound care applications.
\end{abstract}

\footnotetext{
" Corresponding author details: Prof. Ming-Wei Chang; E-mail: mwchang@zju.edu.cn. Tel: +86 (0)57187951517. Fax: +86 (0)571-87951517.
} 


\section{Introduction}

Medical dressings have been widely used to promote healing of various wound types such as venous ulcers ${ }^{1,2}$ and skin burns ${ }^{3,4}$. Traditional medical dressing systems, including cottons, bandages and gauzes, generally provide only simplistic functions ${ }^{5}$. In most cases these need to be used in conjunction with medicated creams or liquids to provide therapeutic benefits or accelerate wound healing. In view of this, various engineering methods have been used to develop novel medical dressings in the last two decades; each with specific advantages as well as a clear focus on the desired scope of application. For example, bandages possessing micro-porous structures have been prepared using freeze-drying and are favorable for exudate absorption from wound surfaces ${ }^{6}$. In addition, electrospun membranes can effectively control release rate of loaded nitric oxide (NO) which is known to contribute towards improved wound healing ${ }^{7}$. Several advances have also been made providing additional functionalities towards these systems such as wound monitoring ${ }^{8}$ and enhanced anti-bacterial properties ${ }^{9}$. Since the wound care market contributes to a very large proportion of pharmaceutical device sales and possesses huge development potential (forecast to reach $\$ 22.01$ billion by $2022^{10}$ ), there is ongoing development and newer conceptual designs in this area.

Furthermore, developments in medical dressings prepared from polymer matrix composites containing active pharmaceutical ingredients (APIs) are on the increase ${ }^{11-}$ ${ }^{16}$. In general, API is dispersed in a polymer matrix which forms the base of dressings and provides protection for wounds. Depending on the application, the matrix is 
designed to provide rapid release of API for emergency conditions ${ }^{13}$ or sustained release of API to achieve long-term management (e.g. of chronic wounds, such as leg ulcers ${ }^{15}$ and deep burns ${ }^{16}$ ).

Electrospinning (ES) is a facile method to produce fibrous materials with predetermined physical and chemical properties by adjusting reaction and process parameters. Furthermore, electrospun materials have been explored in electronics, materials science and biomedicine due to facile structure engineering and related performance ${ }^{17,18}$. In recent times, the engineering of advanced electrospun fibers with greater alignment, tailored surface morphology and compartmentalized internal structures has been shown. This has been achieved using several core and peripheral components including coaxial nozzles ${ }^{19}$, side-by-side channels ${ }^{20}$ and tip collectors ${ }^{21}$. In addition, ES can be used to prepare stable and uniform composite materials with enhanced properties. For example, fillers with excellent thermal properties have been dispersed consistently in fibers through the ES technique to improve material properties of composites ${ }^{22,23}$.

Electrospraying (ESy) is another flexible electrohydrodynamic technique that has been used to produce micro- and nano-scaled particles ${ }^{24,25}$. Applications involving active incorporated structures have shown improved cellular uptake ${ }^{26}$, enhanced intestinal permeability ${ }^{27}$, increased oral bioavailability ${ }^{28}$ and several other advantages as drug delivery dosage forms ${ }^{29}$. The ESy process is similar to ES but with differences to jet break-up and droplet elongation arising from physical properties of the liquid medium (or formulation) used ${ }^{30}$. Previous studies have shown combinatorial 
approaches; where both ES and ESy techniques have been used (simultaneously or one directly after the other) to achieve complex 3D structures. These include bead embedded nanofibers for scaffolds ${ }^{31}$, nanoparticle-loaded polyimide matrices ${ }^{32}$ and honeycomb-like fibrous materials ${ }^{33}$. Furthermore, by utilizing physical and chemical properties of materials; resulting micron-scaled architectures provide timely developments for cell growth ${ }^{31}$, capacitors ${ }^{32}$ and tissue regeneration ${ }^{34}$.

Thermoplastic polyurethane (TPU) is a class of thermoplastic elastomer, which has been used to develop several composite biomaterials because of its excellent mechanical properties, biocompatibility and blood compatibility ${ }^{35,36}$. Poly( $(\varepsilon-$ caprolactone) (PCL) is a linear aliphatic biocompatible polyester, which has been widely used in the medical field for numerous applications, including pharmaceutical dosage form development ${ }^{37,38}$ and cardiovascular stents ${ }^{39,40}$. For drug delivery, PCL is often used as a matrix material to prolong active release ${ }^{41-44}$. Moreover, when compared to other common biocompatible polymers, such as PLA, PCL has higher drug permeation ${ }^{45}$, better patient compliant feel ${ }^{46}$, enhanced degradation times ${ }^{47}$ as well as economic factors.

In this study, elastic anti-bacterial hybrid membranes (HMs) were prepared as novel wound healing composite materials using a combination of ES and ESy techniques. Three TPU fibrous membranes (FMs) were prepared as dressing support materials (providing elasticity) using a rotating collector. Tetracycline hydrochloride (TE-HCL) was loaded into PCL microparticles (MPs) as matrix based controlled release components. Optical microscopy (OM), scanning electron microscopy (SEM) and 
Fourier Transform infrared (FTIR) spectroscopy were used to study morphology and chemical components of various HMs. The developed elastic anti-bacterial hybrid membranes show potential application in wound healing, since the hydrophilic inner layer is capable of absorbing exudate and keep the wound site clean, while hydrophobic outer layer provides waterproofing ability. In addition, mechanical properties and TEHCL antibiotic release can be controlled for long-term use and facilitate recovery at the wound site through geometrical fiber arrangement. Finally using fiber and particle laden system ensures the system provides breathability which would also expedite wound healing.

\section{Experimental Section}

\subsection{Materials}

PCL (mean $M_{\mathrm{w}}=4.5 \times 10^{4} \mathrm{~g} / \mathrm{mol}$ ) was purchased from Sigma-Aldrich Ltd., St Louis, USA. TPU (mean $M_{\mathrm{w}}=2.0 \times 10^{4} \mathrm{~g} / \mathrm{mol}$ ) was provided by Solvay China Ltd., Shanghai, China. Glacial acetic acid (HAc, $99.5 \%$ ), N,N-Dimethylformamide (DMF, $99.8 \%$ ) and Phosphate-buffered saline (PBS, $\mathrm{pH}=7.4$ ) were obtained from Sinopharm Chemical Reagent Co., Ltd., Shanghai, China. TE-HCL was supplied by Amresco Inc., USA. Ultrapure water was prepared using a Millipore Milli-Q Reference ultrapure water purifier (USA). All materials were of the analytical grade and used without further purification.

Escherichia coli (E. coli) was purchased from Maojie Microbial Sci. \& Tech. Co., Ltd., Nanjing, China. Staphylococcus aureus (S. aureus) was supplied by National 
Center for Medical Culture Collections, Beijing, China. Violet Red Bile agar and BairdParker agar were obtained from Hope Bio-Technogy Co., Ltd., Qingdao, China.

\subsection{Preparation of PCL and TPU solutions}

$6 \mathrm{wt} \%$ PCL solution was prepared by dissolving PCL in HAc. TE-HCL was selected as a model antibiotic and was subsequently added to the PCL solution at 0.48 $\mathrm{wt} \%$. $20 \mathrm{wt} \%$ TPU solution was prepared by dissolving the polymer in DMF. Each initial mixture was subjected to magnetic stirring (VELP ARE heating magnetic stirrer, Italy) continuously at $300 \mathrm{rpm}$ for $4 \mathrm{~h}$ at the ambient temperature $\left(25^{\circ} \mathrm{C}\right)$ to ensure complete dissolution.

\subsection{Fabrication of hybrid membranes (HMs)}

Figure 1 shows experimental set-up which comprises high voltage power supply (Glassman high voltage Inc. series FC, USA), a syringe pump (KD Scientific KDS100, USA), a rotating drum and a stainless steel needle system. The needle (inner and outer diameters: 0.20 and $0.24 \mathrm{~mm}$, respectively) was connected to the high voltage power supply and was positioned directly above the earth-grounded rotating drum (diameter and length 7 and $14 \mathrm{~cm}$ (respectively), rotation speed variable: 0-4000 rpm). The rotating drum was layered with Aluminum foil for ease of removal post deposition.

In the first step, a TPU solution was infused from a plastic syringe into the needle inlet via the syringe pump. TPU fibers were fabricated using ES whilst maintaining a flow rate and an applied voltage at $0.5 \mathrm{~mL} / \mathrm{h}$ and $12 \mathrm{kV}$, respectively. The distance 
between needle outlet and rotating drum was set to $11 \mathrm{~cm}$. The rotation speed of the collector was varied from 200 to $3200 \mathrm{rpm}$ during this step for preparing TPU FMs with three well defined patterns. Samples were collected for 20 mins and subsequently analyzed using microscopy. TPU FMs consisting of random (TPU FMs (R)) and parallel fibers (TPU FMs (P)) were prepared at $200 \mathrm{rpm}$ and $3200 \mathrm{rpm}$, respectively. TPU FMs consisting of vertical fibers (TPU FMs (V)) were prepared by repeating TPU FMs (P) fabrication step four times. At designated time intervals (5 mins) deposited TPU fibers were repositioned $90^{\circ}$ clockwise every two times. An extended collection time (4 h) was used to prepare samples for coating membranes with PCL MPs in the next step.

The second step was to load a PCL solution into a separate plastic syringe at a flow rate of $1.0 \mathrm{~mL} / \mathrm{h}$. The distance between needle outlet and rotating drum was maintained at $9 \mathrm{~cm}$ and an applied voltage was kept at $9 \mathrm{kV}$. PCL MPs were fabricated using ESy and the collection time was $3 \mathrm{~h}$. The rotation speed was set at $200 \mathrm{rpm}$ to ensure equal distribution over membranes. MPs were collected directly on to TPU FMs. The resulting HMs comprising both MPs and FMs were termed HMs (R), HMs (P) and HMs (V), respectively (Table 1). All experiments were performed at ambient temperature $\left(25^{\circ} \mathrm{C}\right)$. Finally, HMs were removed from the collector and dried in air at $12 \mathrm{~h}$ before characterization.

\subsection{Characterization}

Optical microscopy (OM, Phenix BMC503-ICCF, China) and scanning electron 
microscopy (SEM, SU 8000 SEM, Hitachi, Japan) analyses were performed to study morphology of PCL MPs, TPU FMs and HMs. For SEM analysis, samples were observed at an accelerating voltage of $20 \mathrm{kV}$. Prior to analysis, samples were sputtercoated (Ion Sputter MC 1000, Hitachi, Japan) with a thin layer of gold for $120 \mathrm{~s}$ at a current intensity of $25 \mathrm{~mA}$. Fourier Transform infrared spectroscopy (FTIR, IR Affinity1, Shimadzu, Japan) was performed to determine the chemical composition of samples. Prior to analysis, samples were prepared by the $\mathrm{KBr}$ pellet method: $2 \mathrm{mg}$ of each sample (pure PCL particles, pure TE-HCL powder, TE-HCL-loaded PCL MPs, TPU FMs and HMs) was mixed with $200 \mathrm{mg}$ of $\mathrm{KBr}$ medium, and then the mixture was compressed into a pellet at $20 \mathrm{MPa}$ for $120 \mathrm{~s}$, respectively. Spectra were recorded from 3500 to $500 \mathrm{~cm}^{-1}$ at a resolution of $4 \mathrm{~cm}^{-1}$.

\subsection{Water contact angle measurements}

An optical contact angle and interface tension meter (SL200KB, KINO Industry Co. Ltd., USA) was used to measure water contact angles for determining hydrophobic/hydrophilic nature of membrane surfaces. An ultrapure water droplet $(\sim 10$ $\mu \mathrm{L})$ was dripped onto each sample, and then values of contact angle were calculated and recorded automatically. For each sample, measurements were taken in triplicate.

\subsection{Tensile test}

A universal materials tester (Zwick/Roell Z020, Zwick, Germany) was used to measure mechanical properties of HMs. Prior to analysis, $40 \times 10 \mathrm{~mm}^{2}$ sized samples 
were prepared. Assessment was initiated without preload and samples were extended with a $500 \mathrm{~N}$ load cell at a stretching speed of $10 \mathrm{~mm} / \mathrm{min}$. For HMs $(\mathrm{P})$, the tensile test direction was along the fiber axis. For each sample type, measurements were taken in triplicate.

\subsection{In vitro drug release test}

Each sample constituting $200 \mathrm{mg} \mathrm{HMs}$ was placed into a glass vial with $10 \mathrm{~mL}$ PBS $(\mathrm{pH}=7.4)$ as the release medium ${ }^{43}$. TE-HCL release was investigated using UV spectrophotometry (Shimadzu, Japan) recorded from 200 to $800 \mathrm{~nm}$ over a $120 \mathrm{~h}$ test period at the ambient temperature $\left(25^{\circ} \mathrm{C}\right)$. At each designated time interval, $4 \mathrm{~mL}$ of supernatant was removed, and the vial was replenished with $4 \mathrm{~mL}$ of fresh PBS. An absorbance (wavelength $=365 \mathrm{~nm}$ ) peak was selected to determine concentration of TE-HCL following calibration. For each sample type experiments were performed in triplicate.

The quantity of encapsulated TE-HCL was calculated using concentration measurements. The weight of PCL MPs and added content of TE-HCL were calculated based on flow rate and collection time.

The LC (loading capacity) of TE-HCL in PCL MPs was calculated using Eq. (1):

$$
\mathrm{LC}(\%)=\frac{\text { weight of encapsulated } T E-H C L}{\text { weight of } P C L M P S} \times 100 \%
$$

The EE (encapsulation efficiency) of TE-HCL in PCL MPs was calculated by Eq. (2): 


$$
\mathrm{EE}(\%)=\frac{\text { weight of encapsulated } T E-H C L}{\text { weight of initial added } T E-H C L} \times 100 \%
$$

\subsection{Anti-bacterial activity test}

The inhibition zone for each membrane sample was measured to determine its antibacterial properties using the agar diffusion method ${ }^{48}$. E. coli was used as representative Gram-negative (G-) bacteria while S. aureus was selected as model Gram-positive $\left(\mathrm{G}^{+}\right)$bacteria. Prior to plating bacteria, $20 \mathrm{~mL}$ of Violet Red Bile Agar and Baird-Parker Agar was poured into sterile petri dishes, respectively. After solidification, $200 \mu \mathrm{L}$ E. coli suspension was spread on to Violet Red Bile Agar plates while $200 \mu \mathrm{L}$ of $S$. aureus suspension was spread on to Baird-Parker Agar plates. Selected fabricated membranes (as $4 \mathrm{~mm}$ diameter discs) were placed on both plates. The plates were incubated at $37{ }^{\circ} \mathrm{C}$ for $24 \mathrm{~h}$. The bacterial inhibition zone of each sample was measured 20 times using ImageJ software and data is presented as the mean diameter of the disc inhibition zone.

\section{Results and Discussion}

\subsection{Fabrication of hybrid membranes (HMs)}

For the ES process, TPU solution was stretched into a narrow and stable Taylor cone at the tip of the nozzle arising due to an inter-balance between electrical stresses and liquid surface tension ${ }^{49}$, which subsequently led to the formation of TPU fibers. Furthermore, through the use of a rotating collector system, such as a rotating drum or a disc, the acquisitions of further stretched and highly oriented fibers have been reported 
${ }^{50-52}$. Compared to a rotating disc, fibers can be prepared in larger quantities when using a drum because of a larger deposition area, which therefore improves efficiency in production rate and also spatial control. With the aid of the collector, two rotation speeds (200 and $3200 \mathrm{rpm}$ ) as well as two forming methods (ES and ESy), TPU FMs with three different structural designs were prepared by alternating fiber deposition angle (at $90^{\circ}$ ).

The ESy process (to yield PCL MPs) was identical to ES in terms of the applied apparatus and method, although differences in product structure arise due to medium properties such as a polymer concentration and chain behavior in solution. Prior to collecting PCL MPs on TPU FMs, a sample of MPs were deposited on to microscopic glass slides for OM. By combining ES and ESy techniques via side-by-side arrangement, three types of HMs were prepared. Fabrication proceeded by patterning of TPU FMs with designated alignment after which membranes were laden with PCL MPs hosting TE-HCL via ESy, as shown in Figure 1.

\subsection{Morphology of synthesized particles and fibers}

Row 1 (Figure 2a, d, $\mathbf{g}, \mathbf{j}, \mathbf{m}, \mathbf{p}$ and $\mathbf{s}$ ) shows schematic diagrams of PCL MPs, TPU FMs [(R), (P) and $(\mathrm{V})]$ and HMs $[(\mathrm{R}),(\mathrm{P})$ and $(\mathrm{V})]$. Corresponding rows 2 (Figure $\mathbf{2 b}, \mathbf{e}, \mathbf{h}, \mathbf{k}, \mathbf{n}, \mathbf{q}$ and $\mathbf{t}$ ) and $\mathbf{3}$ (Figure $\mathbf{2 c}, \mathbf{f}, \mathbf{i}, \mathbf{l}, \mathbf{o}, \mathbf{r}$ and $\mathbf{u}$ ) show optical and scanning electron micrographs, respectively.

PCL MPs are near-uniform and spherical (as shown in Figure $\mathbf{2 b}$ and $\mathbf{c}$ ) possessing a mean diameter of $5.1 \pm 0.2 \mu \mathrm{m}$. Product uniformity proved the process to be suitable 
for mass and stable particle production which is important for industrial applications. Previous studies have shown that spherical PCL particles are ideal for sustained and controlled drug release ${ }^{44,53}$.

TPU fiber alignment was varied from random to ordered as shown in Figure 2d-l. In the first instance, the rotating drum was used to collect TPU fibers at different rotating speeds (from 200 to $3200 \mathrm{rpm}$ ) and study its impact on fiber deposition. At relatively low speeds $(<200 \mathrm{rpm})$, fibers appear randomly orientated (Figure 2d, e and f) with no regular arrangement. Increasing the rotation speed is conducive to fiber orientation control. The ratio of well aligned fibers increases as the rotation speed is increased and appeared most ordered at the highest rotation speed of $3200 \mathrm{rpm}$ (Figure $\mathbf{2 g}, \mathbf{h}$ and i). during this process, the right compromise between rotation speed and evaporation rate of solvent is crucial for the formation of highly oriented fibers ${ }^{54}$. When the collector rotation speed $<<$ fiber deposition speed, fibers appeared randomly overlapped and solvent evaporation is hindered. When the rotation speed is $>>$ fiber deposition speed; continuous fiber collection is difficult due to breakage caused by finer stretched fibers. Therefore, using the rotation speed as a controlled deposition parameter, random and parallel TPU fibers were prepared. Post 4 h' collection, TPU FMs (R) and TPU FMs (P) were collected for assessment. Vertical TPU fibers were prepared by repositioning parallel fibers $\left(90^{\circ}\right)$ thrice (Figure $\mathbf{2 j}$ ). Figure $\mathbf{2 k}$ and $\mathbf{I}$ show optical and electron micrographs of TPU fibers deposited in grid patterns, respectively. Whilst each deposition step was $1 \mathrm{~h}$, FMs (V) were prepared with four layers at a stack angle of $90^{\circ}$. Diameters of TPU fibers in FMs $(\mathrm{R}),(\mathrm{P})$ and $(\mathrm{V})$ were $1.21 \pm 0.22,1.06 \pm 0.16$ and 
$1.02 \pm 0.21 \mu \mathrm{m}$, respectively. The results show that there was no significant difference in the fiber diameter between FMs (P) and (V) due to deployment of identical parameters. However, fiber stretching due to accelerated rotation speeds, leads to reduced diameters and greater uniformity compared to FMs (R).

Once optimized, TPU FMs were transferred to the adjacent ESy nozzle for PCL MPs deposition. Each membrane type was deposited with MPs for $3 \mathrm{~h}$ leading to the formation of HMs (R) (Figure 2n), HMs (P) (Figure 2q) and HMs (V) (Figure 2t).

\subsection{Water contact angle measurements}

Water contact angle measurements elucidate the hydrophobic/hydrophilic nature of a materials surface. The results of water contact angle on three FMs and corresponding HMs are shown in Figure 3. The surfaces for all TPU samples indicate FMs are hydrophobic. The mean water contact angle on TPU FMs $(\mathrm{R})$ is $116.93 \pm 0.49^{\circ}$, which was the most pronounced among all samples. TPU FMs (P) and (V) exhibit water contact angles of $114.92 \pm 0.29^{\circ}$ and $110.14 \pm 0.73^{\circ}$, respectively.

The hydrophobicity of membrane surface is largely driven by material composition. TPUs comprise segmented polymeric chains composed of hard and soft blocks, and the ratio between hard and soft block determines interfacing properties ${ }^{55}$. When hydrophobic hard segments play a more dominant role than hydrophilic soft segments, water contact angles are more likely to be greater than $90^{\circ} 56$. In addition, fiber alignment also impacts surface hydrophilicity. Compared to TPU FMs (R), the slight increase in surface hydrophilicity is due to an increase in voids for TPU FMs (P) and 
(V) ${ }^{54,55}$ thus contributing to larger pore volume in FMs.

PCL MP deposition modifies the hydrophobicity of TPU FMs. The results indicate surfaces incorporating PCL MPs are hydrophilic, with TPU FMs (R), (P) and (V) possessing water contact angles of $78.15 \pm 3.15^{\circ}, 61.56 \pm 6.37^{\circ}$ and $67.14 \pm 2.98^{\circ}$, respectively. The clear difference in hydrophilic nature arises due to factors relating TEHCL-loaded PCL MPs. Although PCL is hydrophobic, PCL MP deposition significantly increases surface roughness of membranes which is known to impact water contact angle ${ }^{48}$. Hence, the hydrophilic nature of HMs provides an opportunity to absorb exudate and thus keep the wound site clean. In addition, the reverse side of HMs remain hydrophobic; providing waterproofing and barrier properties.

\subsection{Tensile test}

Mechanical properties of HMs were evaluated using tensile testing. Test results for three selected samples characterized by stress-strain curves, are shown in Figure 4. Among these samples, HMs (P) displayed the greatest tensile strength $(32.1 \pm 4.9 \mathrm{MPa})$. As the alignment of TPU fibers decreases, the tensile strength of HMs (V) and (R) declines; exhibiting tensile strength values of $12.2 \pm 2.1$ and $11.6 \pm 1.6 \mathrm{MPa}$, respectively. However, tensile strain was seen to increase as the alignment decreased. The tensile strain for HMs (P) was the lowest (104.2 $\pm 6.0 \%)$ with HMs (R) displaying the greatest strain value $(225.6 \pm 7.3 \%)$.

Tensile properties of HMs are affected by fiber microstructure, individual fiber properties and geometrical arrangement ${ }^{54}$. The three FMs have a near identical 
microstructure based on the TPU polymer. Fiber diameter has an appreciable influence on tensile properties of individual fibers. Three geometrical arrangements are shown in Figure 2. The tensile strength decreases with increasing fiber diameter ${ }^{57,58}$. Also, well aligned fibers display greater tensile strength when compared to their randomly arranged counterparts ${ }^{59}$.

For HMs (R), random fiber arrangement, with fibers possessing large diameters, ensures interconnection and multi-directional integration; not favoring tensile strain enhancement for a specific direction. For this reason, $\mathrm{HM}(\mathrm{R})$ membranes displayed the greatest tensile strain as well as the lowest tensile strength. Tensile strength was simplified equivalent to the product of fiber numbers and the average strength vector along the tensile direction of a single fiber, which led to the lowest tensile strength among three HMs.

For HMs (P), fibers are parallel (adjacent) and the tensile direction is along the fiber axis. Compared to HMs (R), greater fiber alignment enables HMs (P) to display the highest tensile strength although accompanied by reduced elasticity. In this regard, elastic HMs (P) with high tensile strength indicate the materials suitability as a medical membrane material. In addition, the stress-strain curve for HMs (V) show two tensile values. This phenomenon arises because of deposited fiber arrangement. During local plastic deformation stage, when the external force exceeds strength limit, the first layer shrinks, leading to a decrease in strength until breakage. The remaining three layers consolidate the strengthening stage which leads to a secondary local plastic deformation stage, and the sample totally breaks at the second fracture point. 


\subsection{FTIR analysis}

Figure 5 shows the FTIR spectra of pure PCL particles, pure TE-HCL powder, TEHCL-loaded PCL MPs, TPU FMs and HMs, focusing in the 3500 to $500 \mathrm{~cm}^{-1}$. For PCL, characteristic peaks at $1734 \mathrm{~cm}^{-1}, 2950 \mathrm{~cm}^{-1}$ and $2863 \mathrm{~cm}^{-1}$ indicate $\mathrm{C}=\mathrm{O}$ stretching, $\mathrm{CH}_{2}$ asymmetric stretching and symmetric stretching, respectively ${ }^{60}$. For TE-HCL, the main peak at $1675 \mathrm{~cm}^{-1}$ shows the presence of $\mathrm{C}=\mathrm{O}^{61}$. For TPU, the peak at $1521 \mathrm{~cm}^{-1}$ represents amide $\mathrm{II}^{56}$. Compared to PCL, a new peak at $1676 \mathrm{~cm}^{-1}$ is observed which belongs to TE-HCL for TE-HCL-loaded PCL MPs. All aforementioned characteristic peaks appear in TE-HCL/PCL/TPU, which confirms HMs were prepared successfully without any interactions between various components.

\subsection{In vitro drug release}

LC and EE of TE-HCL in PCL MPs was $5.78 \%$ and $86.72 \%$, respectively. In vitro drug release profiles are shown in Figure 6. A variation in TE-HCL release from three HMs was observed. HMs (R) samples exhibit fastest cumulative drug release while HMs (P) samples display the most sustained release. However, compared to HMs (P) and (V); HMs (R) samples display a rapid initial release phase $(0-2 \mathrm{~h})$. For example, after $1 \mathrm{~h}$, the cumulative release ratios of TE-HCL from HMs (R), (V) and (P) were $67.52 \%, 43.42 \%$ and $38.74 \%$, respectively. The drug release process includes two main steps; particle detachment from membranes and drug diffusion from MPs.

An important factor contributing towards release rate relates to pore or void 
properties for each sample, which vary due to geometrical fiber arrangement. TPU FMs (R) were prepared by collecting fibers randomly and have minimum voidage because of dense deposition in multiple directions, as shown in Figure 2o. According to previous studies ${ }^{62}$ particles merging with other particles exhibits greater release rates. With dense randomly distributed fibers, voids are reduced and particle merging is favored. However, for TPU FMs (V) maximum void volume can be expected because of the well-placed stacked structure, as shown in Figure $\mathbf{2 u}$. Here, drug-loaded particles are able to deposit directly into pores and onto struts. Void volume for TPU FMs (P) is between the previously described (two) sample types. Here, most particles are embedded in crevices between parallel fibers, resulting in an increase in particlemembrane forming. Consequently, HMs (P) display the most sustained release rate. In conclusion, in vitro drug release tests show geometrical arrangement of membrane contributing fibers has a great impact on drug release performance.

\subsection{Anti-bacterial test}

Wound healing management involves the prevention or treatment of bacterial infections ${ }^{5}$. TE-HCL was selected as a model drug based on the broad spectrum antibacterial ability ${ }^{63,64}$. The agar diffusion method was used to evaluate anti-bacterial properties of HMs over a $24 \mathrm{~h}$ test period. E. coli and $S$. aureus were used as model Gram-negative (G-) and Gram-positive (G+) bacteria, respectively. The inhibition zone of each sample is shown in Figure 7a and corresponding measurements are shown in Figure $7 \mathbf{b}$. 
Figure 7a (I) and (VI) show Violet Red Bile agar and Baird-Parker agar plates without bacteria, respectively. After E. coli and S. aureus suspensions were spread on to corresponding plates and incubated for $24 \mathrm{~h}$; color change on both plates was evident, as shown in Figure 7a (II) and (VII). Three types of HMs discs (diameter $\sim 4 \mathrm{~mm}$ ) were used for bacterial assessment. Figure 7a (III) to (V) show inhibition zones arising from HMs (R), (P) and (V) discs against E. coli exhibiting diameters of $18.28 \pm 0.16 \mathrm{~mm}$, $18.23 \pm 0.03 \mathrm{~mm}, 17.05 \pm 0.24 \mathrm{~mm}$, respectively. Figure 7a (VIII) to (X) show inhibition zones against $S$. aureus displaying inhibition zones of $18.26 \pm 0.14 \mathrm{~mm}$, $18.10 \pm 0.14 \mathrm{~mm}, 18.18 \pm 0.14 \mathrm{~mm}$, respectively. The results indicate matrices are ideal to prevent bacterial activity when compared to control groups (Figure 7a (II) and (VII)). Inhibition zones displayed slightly improved activity at the $24 \mathrm{~h}$ point. The results indicate matrices are well suited to prevent bacterial activity when compared to control groups (Figure 7a (II) and (VII)). In addition, an insignificant difference between inhibition zones in three experimental groups shows that fiber direction does not impact E. coli and $S$. aureus inhibition. This further confirms drug release from TE-HCL loaded PCL particles has a great impact on anti-bacterial performance.

\section{Conclusion}

Medical bandages are vital to wound healing and repair. Thus, developing a facile method to fabricate bandages with tailored strength, anti-bacterial and managed drug release properties becomes imperative. In our work, PCL MP modified TPU FMs with three different fibrous patterns ( $\mathrm{R}, \mathrm{P}$ and $\mathrm{V})$ were fabricated combining ES with ESy 
techniques. TE-HCL was loaded in PCL MPs as a model drug, and TPU FMs patterning was regulated using a rotating collector. OM, SEM and FTIR analyses indicate three patterns were successfully prepared. Water contact angle tests show HMs possess a hydrophobic outer layer and hydrophilic inner layer; providing waterproofing and wound exudate absorption properties, respectively. Tensile tests show HMs (P) have high tensile strength with elasticity. In vitro drug release tests also demonstrate HMs (P) have sustained-release properties. Anti-bacterial tests indicate all three HMs have outstanding anti-bacterial activity against E. coli and S. aureus. In summary, HMs (P) are the best candidate for skin wound care applications. Compared to other drug loaded membranes as potential skin products, the current system displays multiple functions with tailorable aspects, which can be prepared in rapid fashion.

\section{Acknowledgements}

This work was financially supported by the National Nature Science Foundation of China (No. 81771960), the Fundamental Research Funds for the Central Universities (2017QNA5017) and Key Technologies R\&D Program of Zhejiang Province (2015C02035).

\section{Conflict of Interest}

The authors declare no conflict of interest.

\section{Keywords}

Hybrid membranes; PCL particles; TPU fibrous membranes; Electrospraying; Electrospinning. 


\section{References}

1. Fletcher, A.; Cullum, N.; Sheldon, T. A., Bmj 315, 5761997.

2. O'Meara, S.; Cullum, N.; Nelson, E. A.; Dumville, J. C., The Cochrane Library2012.

3. Church, D.; Elsayed, S.; Reid, O.; Winston, B.; Lindsay, R., Clinical microbiology reviews 19, 4032006.

4. Mogoşanu, G. D.; Grumezescu, A. M., International journal of pharmaceutics 463, 1272014.

5. Boateng, J. S.; Matthews, K. H.; Stevens, H. N.; Eccleston, G. M., Journal of pharmaceutical sciences 97, 28922008.

6. Kumar, P.; Lakshmanan, V.-K.; Biswas, R.; Nair, S. V.; Jayakumar, R., Journal of biomedical nanotechnology 8, 8912012.

7. Lowe, A.; Bills, J.; Verma, R.; Lavery, L.; Davis, K.; Balkus, K., Acta biomaterialia 13, 1212015.

8. Kassal, P.; Kim, J.; Kumar, R.; de Araujo, W. R.; Steinberg, I. M.; Steinberg, M. D.; Wang, J., Electrochemistry Communications 56, 62015.

9. Sudheesh Kumar, P.; Lakshmanan, V.-K.; Anilkumar, T.; Ramya, C.; Reshmi, P.; Unnikrishnan,

A.; Nair, S. V.; Jayakumar, R., ACS applied materials \& interfaces 4, 26182012.

10. MarketsandMarkets. 2017.

11. Suganya, S.; Senthil Ram, T.; Lakshmi, B.; Giridev, V., Journal of Applied Polymer Science 121, 28932011.

12. Karami, Z.; Rezaeian, I.; Zahedi, P.; Abdollahi, M., Journal of Applied Polymer Science 129, 7562013.

13. Nagy, Z. K.; Balogh, A.; Drávavölgyi, G.; Ferguson, J.; Pataki, H.; Vajna, B.; Marosi, G., Journal of pharmaceutical sciences 102, 5082013.

14. Moritz, S.; Wiegand, C.; Wesarg, F.; Hessler, N.; Müller, F. A.; Kralisch, D.; Hipler, U.-C.;

Fischer, D., International journal of pharmaceutics 471, 452014.

15. Catanzano, O.; Docking, R.; Schofield, P.; Boateng, J., Carbohydrate polymers 172, 402017.

16. Dhand, C.; Venkatesh, M.; Barathi, V. A.; Harini, S.; Bairagi, S.; Leng, E. G. T.;

Muruganandham, N.; Low, K. Z. W.; Fazil, M. H. U. T.; Loh, X. J., Biomaterials 138, 1532017.

17. Huang, Z.-M.; Zhang, Y.-Z.; Kotaki, M.; Ramakrishna, S., Composites science and technology 63, 22232003.

18. Wang, L.; Chang, M.-W.; Ahmad, Z.; Zheng, H.; Li, J.-S., Chemical Engineering Journal 307, 6612017.

19. Yao, Z.-C.; Zhang, C.; Ahmad, Z.; Huang, J.; Li, J.-S.; Chang, M.-W., Chemical Engineering Journal 334, 892018.

20. Lin, T.; Wang, H.; Wang, X., Advanced Materials 17, 26992005.

21. Wang, B.; Zhou, W.; Chang, M. W.; Ahmad, Z.; Li, J. S., Journal of Applied Polymer Science 1342017.

22. Gu, J.; Lv, Z.; Wu, Y.; Guo, Y.; Tian, L.; Qiu, H.; Li, W.; Zhang, Q., Composites Part A: Applied Science and Manufacturing 94, 2092017.

23. Guo, Y.; Xu, G.; Yang, X.; Ruan, K.; Ma, T.; Zhang, Q.; Gu, J.; Wu, Y.; Liu, H.; Guo, Z., Journal of Materials Chemistry C 6, 30042018.

24. Jaworek, A., Powder technology 176, 182007.

25. Gao, Y.; Zhao, D.; Chang, M.-W.; Ahmad, Z.; Li, J.-S., Chemical Engineering Journal 284, 963 2016. 
26. Ding, L.; Lee, T.; Wang, C.-H., Journal of Controlled Release 102, 3952005.

27. Sayed, E.; Karavasili, C.; Ruparelia, K.; Haj-Ahmad, R.; Charalambopoulou, G.; Steriotis, T.; Giasafaki, D.; Cox, P.; Singh, N.; Giassafaki, L.-P. N., Journal of Controlled Release 278, 1422018. 28. Mangrio, F. A.; Dwivedi, P.; Han, S.; Zhao, G.; Gao, D.; Si, T.; Xu, R. X., Molecular pharmaceutics 14, 47252017.

29. Zamani, M.; Prabhakaran, M. P.; Ramakrishna, S., International journal of nanomedicine 8, 29972013.

30. Wang, H.; Liu, Q.; Yang, Q.; Li, Y.; Wang, W.; Sun, L.; Zhang, C.; Li, Y., Journal of materials science 45, 10322010.

31. Li, W.; Li, X.; Wang, T.; Li, X.; Pan, S.; Deng, H., European Polymer Journal 48, 18462012.

32. Ding, Y.; Wu, Q.; Zhao, D.; Ye, W.; Hanif, M.; Hou, H., European Polymer Journal 49, 2567 2013 .

33. Wittmer, C. R.; Hébraud, A.; Nedjari, S.; Schlatter, G., Polymer 55, 57812014.

34. Francis, L.; Venugopal, J.; Prabhakaran, M. P.; Thavasi, V.; Marsano, E.; Ramakrishna, S., Acta biomaterialia 6, 41002010.

35. Huang, C.; Chen, R.; Ke, Q.; Morsi, Y.; Zhang, K.; Mo, X., Colloids and Surfaces B: Biointerfaces 82, 3072011.

36. Lambertz, A.; Vogels, R. R.; Binnebösel, M.; Schöb, D. S.; Kossel, K.; Klinge, U.; Neumann, U. P.; Klink, C. D., Journal of Biomedical Materials Research Part A 103, 26542015.

37. Kweon, H.; Yoo, M. K.; Park, I. K.; Kim, T. H.; Lee, H. C.; Lee, H.-S.; Oh, J.-S.; Akaike, T.; Cho, C.-S., Biomaterials 24, 8012003.

38. Rai, B.; Teoh, S.-H.; Hutmacher, D.; Cao, T.; Ho, K., Biomaterials 26, 37392005.

39. Crowder, S. W.; Gupta, M. K.; Hofmeister, L. H.; Zachman, A. L.; Sung, H.-J., Acta biomaterialia 8, 5592012.

40. Crowder, S. W.; Liang, Y.; Rath, R.; Park, A. M.; Maltais, S.; Pintauro, P. N.; Hofmeister, W.; Lim, C. C.; Wang, X.; Sung, H.-J., Nanomedicine 8, 17632013.

41. Del Valle, L. J.; Camps, R.; Díaz, A.; Franco, L.; Rodríguez-Galán, A.; Puiggalí, J., Journal of polymer research 18, 19032011.

42. Midhun, B.; Shalumon, K.; Manzoor, K.; Jayakumar, R.; Nair, S.; Deepthy, M., Journal of Biomaterials Science, Polymer Edition 22, 24312011.

43. Wang, B.; Zheng, H.; Chang, M.-W.; Ahmad, Z.; Li, J.-S., Colloids and Surfaces B: Biointerfaces 145, 7572016.

44. Zhang, C.; Chang, M.-W.; Li, Y.; Qi, Y.; Wu, J.; Ahmad, Z.; Li, J.-S., RSC Advances 6, 77174 2016.

45. Sinha, V.; Bansal, K.; Kaushik, R.; Kumria, R.; Trehan, A., International journal of pharmaceutics 278, 12004.

46. Scaffaro, R.; Lopresti, F.; Botta, L.; Rigogliuso, S.; Ghersi, G., Journal of the mechanical behavior of biomedical materials 63, 3032016.

47. Simone, E. A.; Dziubla, T. D.; Muzykantov, V. R., Expert opinion on drug delivery 5, 1283 2008.

48. Yao, Z. C.; Chen, S. C.; Ahmad, Z.; Huang, J.; Chang, M. W.; Li, J. S., Journal of Food Science 82, 14122017

49. Taylor, G. Proceedings of the Royal Society of London A: Mathematical, Physical and Engineering Sciences, 1964, pp 383. 
50. Katta, P.; Alessandro, M.; Ramsier, R.; Chase, G., Nano letters 4, 22152004.

51. Yee, W. A.; Nguyen, A. C.; Lee, P. S.; Kotaki, M.; Liu, Y.; Tan, B. T.; Mhaisalkar, S.; Lu, X., Polymer 49, 41962008.

52. Kongkhlang, T.; Tashiro, K.; Kotaki, M.; Chirachanchai, S., Journal of the American Chemical Society 130, 154602008.

53. Gao, Y.; Chang, M.-W.; Ahmad, Z.; Li, J.-S., RSC Advances 6, 881572016.

54. Baji, A.; Mai, Y.-W.; Wong, S.-C.; Abtahi, M.; Chen, P., Composites science and technology 70, 7032010.

55. Mark, H. F., Encyclopedia of polymer science and technology, concise; John Wiley \& Sons, 2013.

56. Mi, H. Y.; Jing, X.; Salick, M. R.; Cordie, T. M.; Peng, X. F.; Turng, L. S., Journal of Biomedical Materials Research Part B: Applied Biomaterials 103, 9602015.

57. Arinstein, A.; Burman, M.; Gendelman, O.; Zussman, E., Nature nanotechnology 2, 592007.

58. Wong, S.-C.; Baji, A.; Leng, S., Polymer 49, 47132008.

59. Huang, C.; Chen, S.; Reneker, D. H.; Lai, C.; Hou, H., Advanced Materials 18, 6682006.

60. Wang, J.-C.; Zheng, H.; Chang, M.-W.; Ahmad, Z.; Li, J.-S., Scientific reports 7, 439242017.

61. Caroni, A.; De Lima, C.; Pereira, M.; Fonseca, J., Colloids and Surfaces B: Biointerfaces 100, 2222012.

62. Li, S.-Q.; Marshall, J., Journal of Aerosol Science 38, 10312007.

63. Zahedi, P.; Karami, Z.; Rezaeian, I.; Jafari, S. H.; Mahdaviani, P.; Abdolghaffari, A. H.; Abdollahi, M., Journal of Applied Polymer Science 124, 41742012.

64. Chen, H.; Xing, X.; Tan, H.; Jia, Y.; Zhou, T.; Chen, Y.; Ling, Z.; Hu, X., Materials Science and Engineering: C 70, 2872017. 
Table 1. HMs Composition.

\begin{tabular}{cc}
\hline Item Name & Description \\
\hline HMs (R) & PCL MPs-TPU FMs (R) \\
HMs (P) & PCL MPs-TPU FMs (P) \\
HMs (V) & PCL MPs-TPU FMs (V) \\
\hline
\end{tabular}




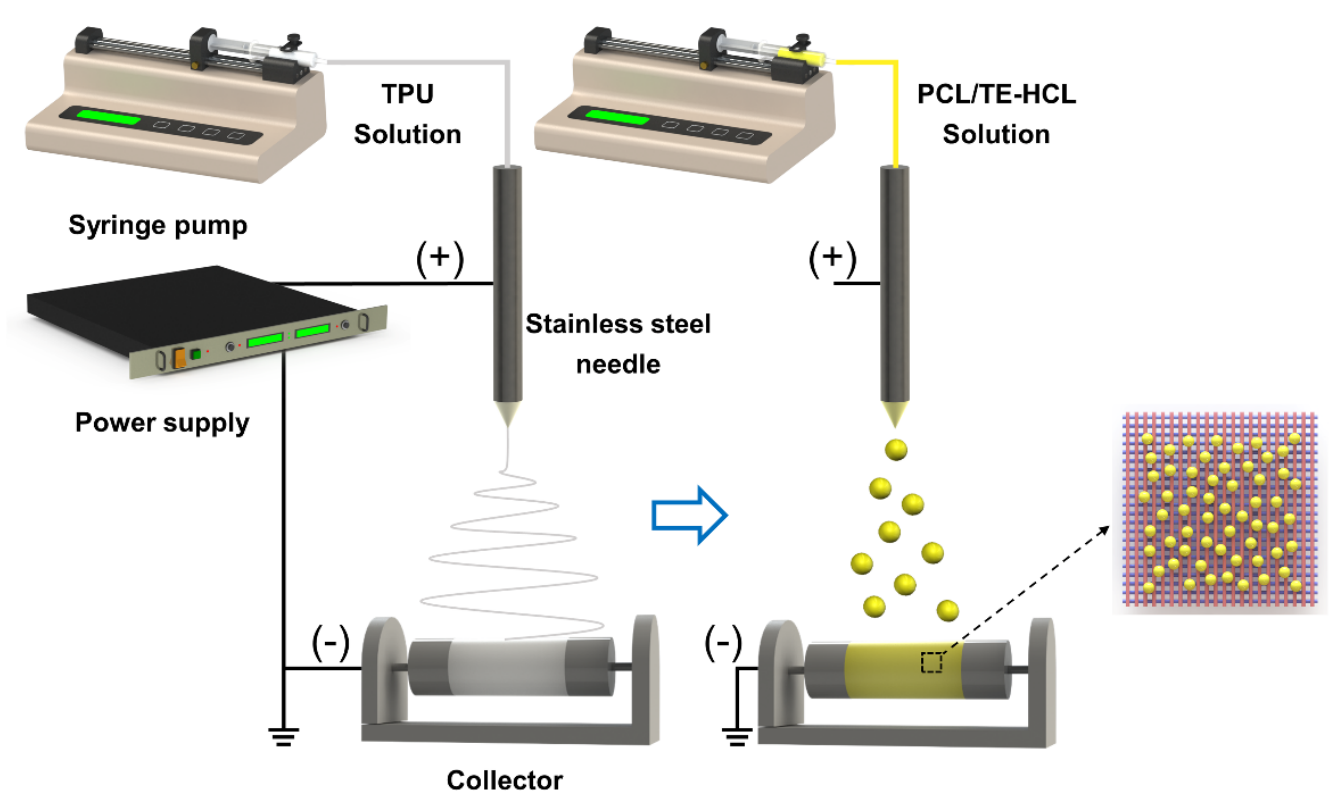

Figure 1. Schematic diagram showing hybrid membrane engineering using ESy and ES techniques via side-by-side approach.

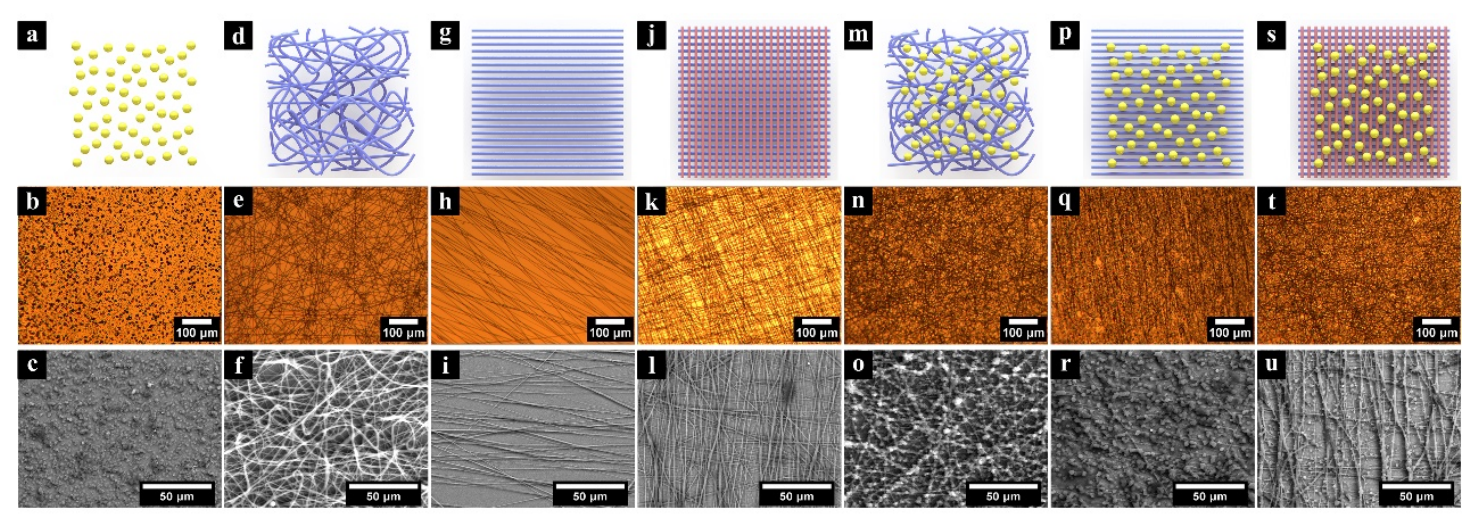

Figure 2. Diagrammatic images, optical and scanning electron micrographs of: (a), (b) and (c) showing PCL MPs. (d), (e) and (f) showing TPU FMs (R). (g), (h) and (i) showing TPU FMs (P). (j), (k) and (l) showing TPU FMs (V). (m), (n) and (o) showing HMs (R). (p), (q) and (r) showing HMs (P). (s), (t) and (u) showing HMs (V). 


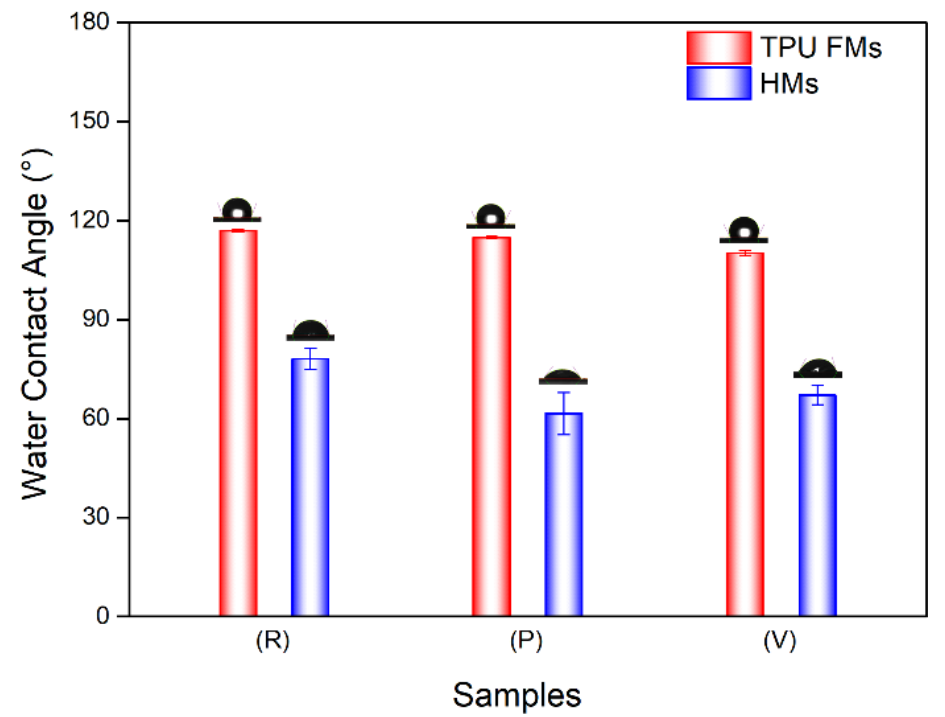

Figure 3. Water contact angles on TPU FMs (R) and HMs (R); TPU FMs (P) and HMs (P); TPU FMs (V) and HMs (V).

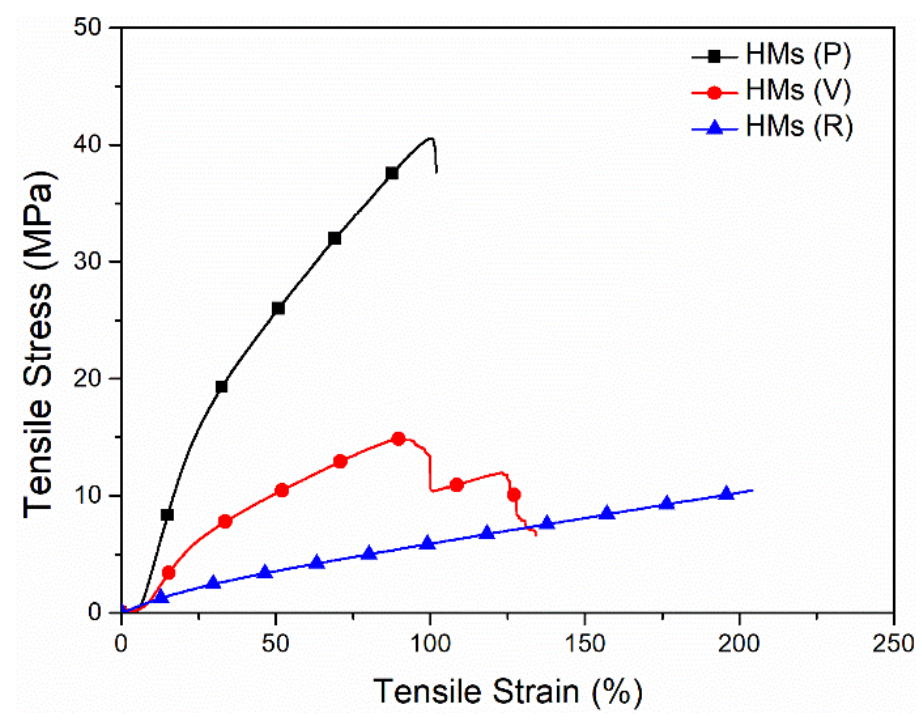

Figure 4. Stress-strain curves of HMs (R), HMs (P) and HMs (V) samples. 


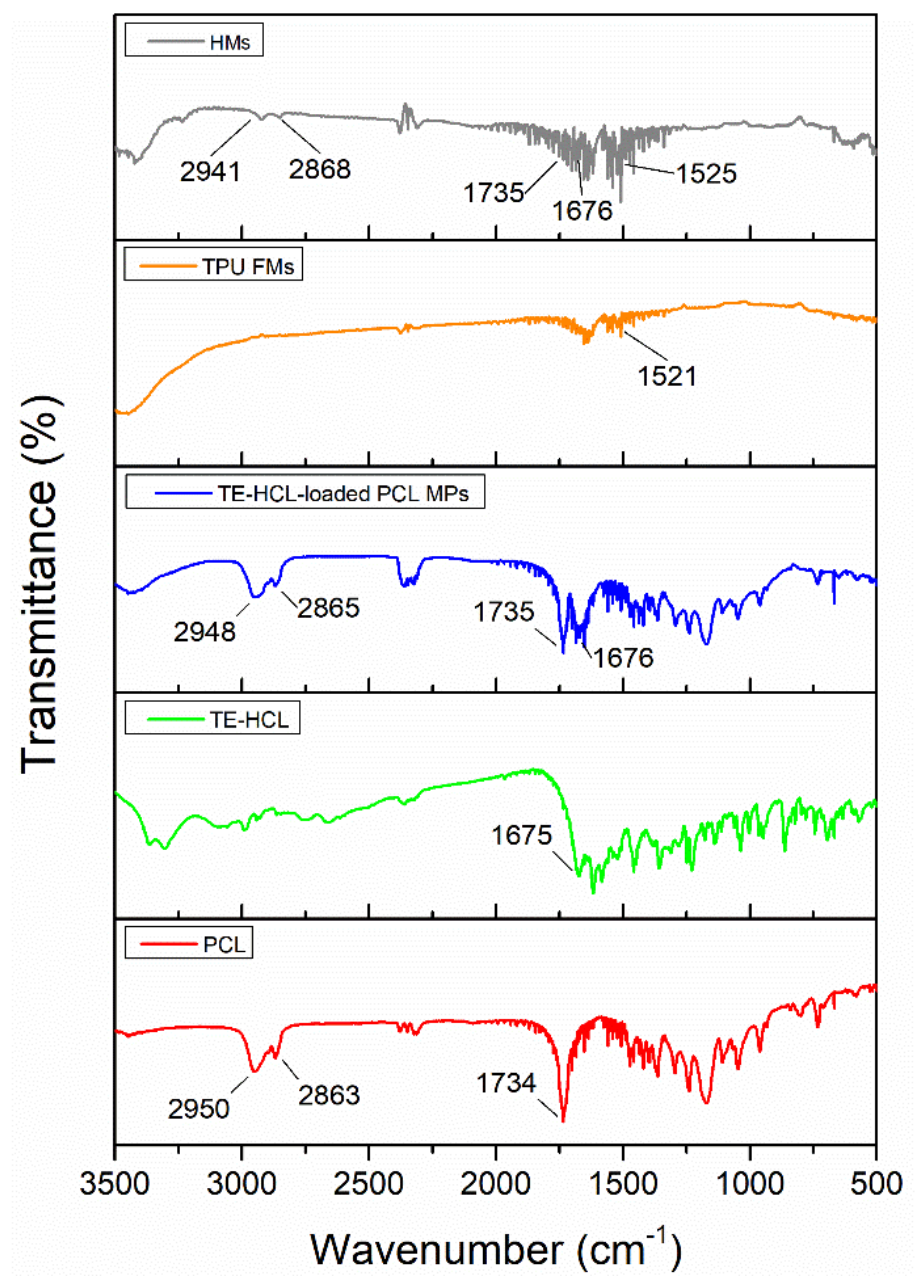

Figure 5. FTIR spectra of HMs, TPU FMs, TE-HCL-loaded PCL MPs, pure TE-HCL powder, pure PCL beads. 


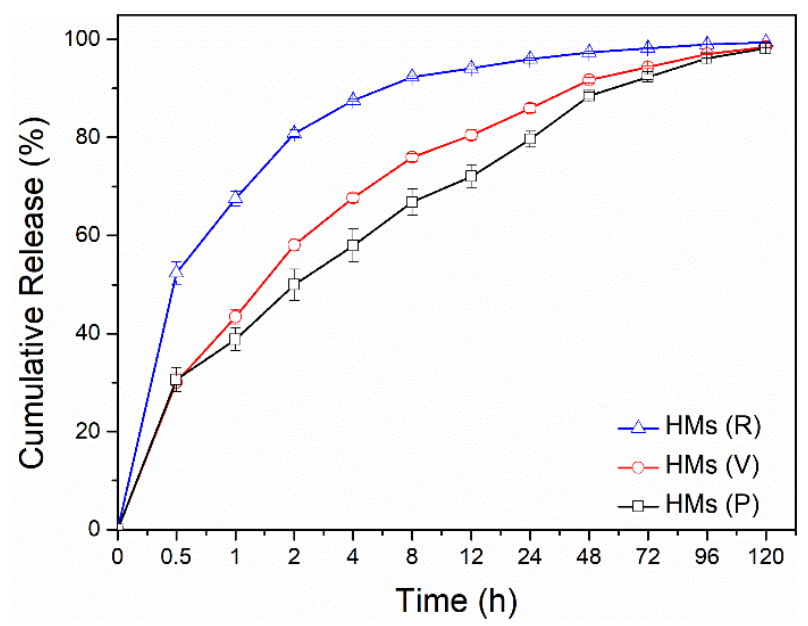

Figure 6. Drug release from HMs (R), HMs (P) and HMs (V) samples.

a

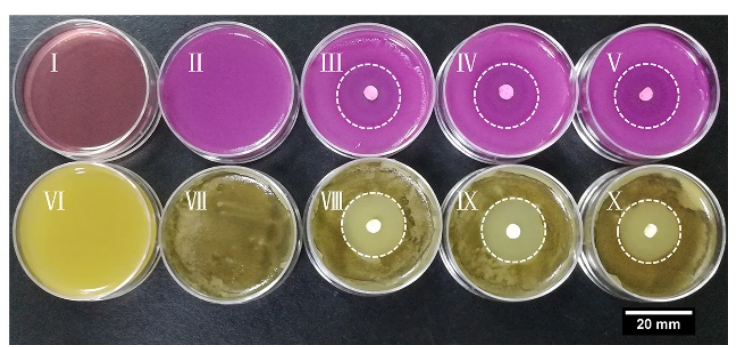

b

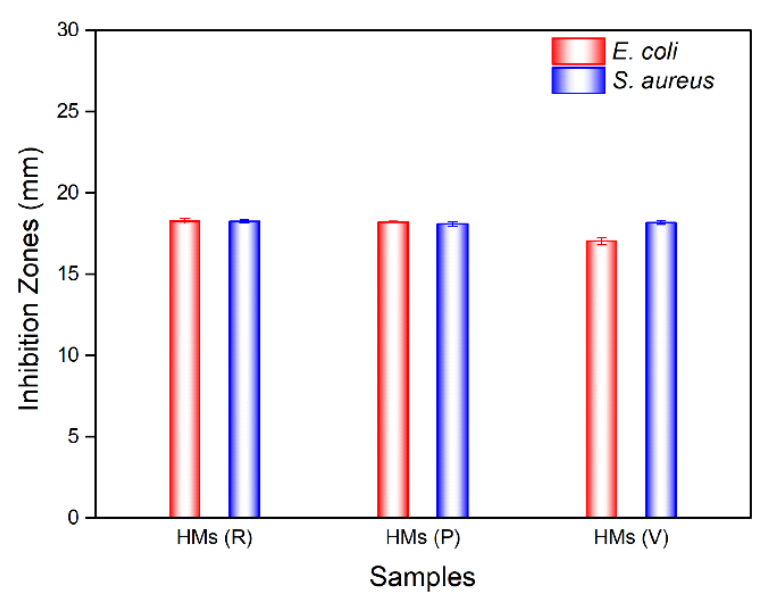

Figure 7. (a) Violet Red Bile agar plates: (I) Blank, (II) E. coli, (III) E. coli with HMs (R) disc, (IV) E. coli with HMs (P) disc and (V) E. coli with HMs (V) disc. BairdParker agar plates: (VI) Blank, (VII) S. aureus, (VIII) S. aureus with HMs (R) disc, (IX) S. aureus with HMs (P) disc and (X) S. aureus with HMs (V) disc. (b) Diameter of inhibition zones of three HMs. 


\section{Text for ToC}

Particulate laden fibrous membranes are prepared using both electrospinning and electrospraying techniques. A rotating collector is used to realize random and ordered fibrous membrane patterns which then display great differences in mechanical and drug release properties.

Figure for ToC_

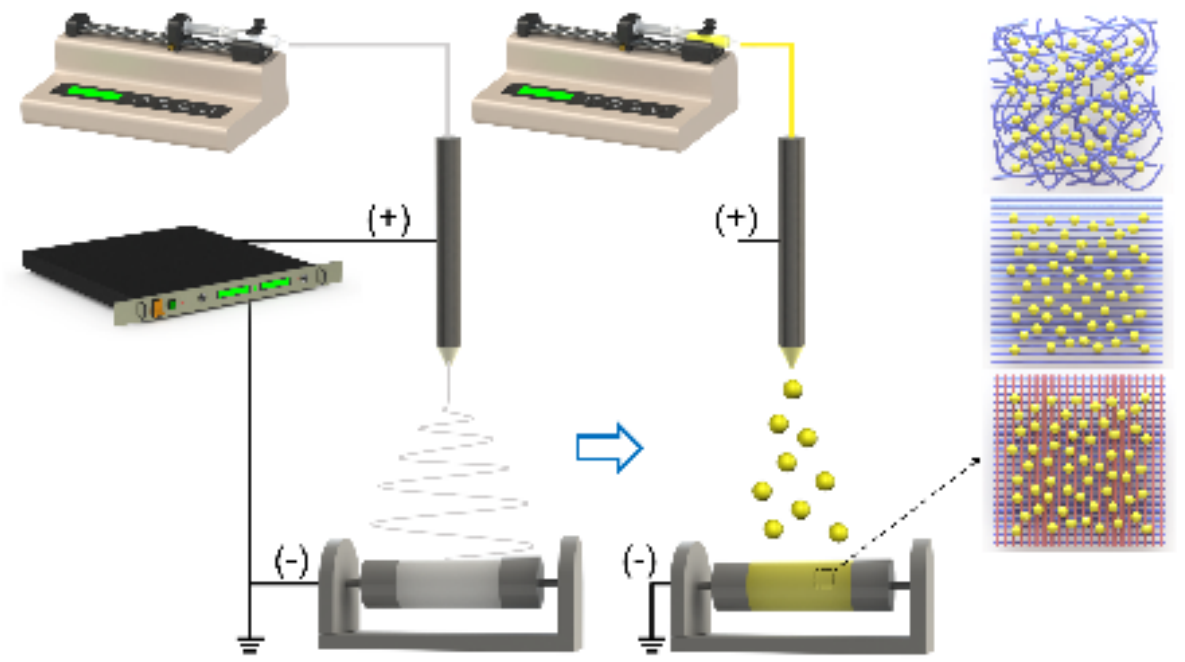

